\title{
ON ALMOST EVERYWHERE CONVERGENCE OF THE GENERALIZED MARCIENKIWICZ MEANS WITH RESPECT TO TWO DIMENSIONAL VILENKIN-LIKE SYSTEMS
}

\author{
GYÖRGY GÁT AND ANTENEH TILAHUN
}

Received 07 October, 2019

Abstract. In this paper we investigate the almost everywhere convergence of two dimensional Marcinkiewicz-like means of two variable integrable functions which is given by

$$
t_{n}^{\alpha} f=\frac{1}{n} \sum_{k=0}^{n-1} S_{\alpha_{1}(|n|, k), \alpha_{2}(|n|, k)} f
$$

$\left(M_{|n|} \leq n<M_{|n|+1}\right)$ and give a sufficient condition for functions $\alpha: N^{2} \mapsto N^{2}$ in order to have the a.e. relation $t_{n}^{\alpha} f \rightarrow f$ for all $f \in L^{1}\left(G_{m}^{2}\right)$ with respect to two dimensional bounded Vilenkin-like systems. Finally, we give an application of the main result with respect to triangular summability of Vilenkin-like-Fourier series.

2010 Mathematics Subject Classification: 42C10

Keywords: almost everywhere convergence, generalized Marcienkiwicz means and two dimensional Vilenkin-like system

\section{INTRODUCTION}

In 1939 for the two-dimensional trigonometric Fourier partial sums $S_{j, j} f$ Marcinkiewicz [14] proved that for all $f \in L \log L\left([0,2 \pi]^{2}\right)$ the a.e. relation

$$
\frac{1}{n} \sum_{j=1}^{n} S_{j, j} f \rightarrow f
$$

holds as $n \rightarrow \infty$. Zhizhiashvili [18] improved this result for $f \in L\left([0,2 \pi]^{2}\right)$. Dyachenko [3] proved this result for dimensions greater than 2. In 2003 Goginava [10] proved this result with respect to the $d$-dimensional Walsh-Paley system. The case $d=2$ is due to Weisz [17]. In 2012 Gát [7] proved this result for generalized Marcinkiewicz means with respect to the two dimensional Walsh system and in 2016 [8] for bounded two-dimensional Vilenkin systems. The aim of this paper is to generalize this result of Gát with respect to two-dimensional generalized Vilenkin-like systems.

The first author is supported by the projects EFOP-3.6.2-16-2017-00015 and EFOP-3.6.1-16-201600022 supported by the European Union, co-financed by the European Social Fund. 
Besides, we give an application of the main result of this paper, that is, Theorem 3 with respect to triangular summability of Vilenkin-Fourier series.

Denote by $\mathbb{N}$ the set of natural numbers and $\mathbb{P}$ the set of positive integers. Denote $m:=\left(m_{k}: k \in \mathbb{N}\right)$ a sequence of positive integers such that $m_{k} \geq 2, k \in \mathbb{N}$ and $G_{m_{k}}$ a set of cardinality $m_{k}$. Suppose that each (coordinate) set has the discrete topology and measure $\mu_{k}$ which maps every singleton of $G_{m_{k}}$ to $\frac{1}{m_{k}}\left(\mu_{k}\left(G_{m_{k}}\right)=1\right), k \in \mathbb{N}$. Let $G_{m}$ be the compact set formed by the complete direct product of $G_{m_{k}}$ with the product of the topologies and measures $(\mu)$. Thus, each $x \in G_{m}$ is a sequence $x:=\left(x_{0}, x_{1}, \ldots\right)$, where $x_{k} \in G_{m_{k}}, k \in \mathbb{N}$. $G_{m}$ is called a Vilenkin space. $G_{m}$ is a compact totally disconnected space, with normalized regular Borel measure $\mu, \mu\left(G_{m}\right)=1$. The Vilenkin space $G_{m}$ is said to be bounded if the generating system $m$ is a bounded one. Throughout this paper the boundedness of $G_{m}$ is supposed. In this paper $C, C_{p}$ denote absolute constants (the latter may depend on $p$ ), which may vary from line to line.

A base for the neighborhoods of $G_{m}$ can be given as follows

$$
I_{0}(x):=G_{m}, \quad I_{n}(x):=\left\{y=\left(y_{i}, i \in \mathbb{N}\right) \in G_{m}: y_{i}=x_{i} \text { for } i<n\right\}
$$

for $x \in G_{m}, n \in \mathbb{P}$.

$$
I:=\left\{I_{n}(x): n \in \mathbb{N}, x \in G_{m}\right\}
$$

is the set of intervals on $G_{m}$.

Denote by $L^{p}\left(G_{m}\right)$ the usual Lebesgue spaces ( $\|\cdot\|_{p}$ the corresponding norms) $(1 \leq$ $p \leq \infty), \mathcal{A}_{n}$ the $\sigma$ algebra generated by the sets $I_{n}(x)\left(x \in G_{m}\right)$ and $E_{n}$ the conditional expectation operator with respect to $\mathcal{A}_{n}(n \in \mathbb{N})$.

Let $L^{0}\left(G_{m}\right)$ be the space of measurable functions on the Vilenkin space $G_{m}$. We say that an operator $T: L^{1}\left(G_{m}\right) \rightarrow L^{0}\left(G_{m}\right)$ is of type $\left(L^{p}, L^{p}\right)$ (for $1 \leq p \leq \infty$ ) if $\|T f\|_{p} \leq C_{p}\|f\|_{p}$ for all $f \in L^{p}\left(G_{m}\right)$, where the constant $C_{p}$ depends only on $p$. We say that $T$ is of weak type $\left(L^{1}, L^{1}\right)$ if $\mu(|T f|>\lambda) \leq C\|f\|_{1} / \lambda$ for all $f \in L^{1}\left(G_{m}\right)$ and $\lambda>0$.

Let $M_{0}:=1$ and $M_{k+1}:=m_{k} M_{k}$, for $k \in \mathbb{N}$ be the so-called generalized powers. Then every $n \in \mathbb{N}$ can be uniquely expressed as $n=\sum_{k=0}^{\infty} n_{k} M_{k}, 0 \leq n_{k}<m_{k}, n_{k} \in \mathbb{N}$. This allows one to say that the sequence $\left(n_{0}, n_{1}, \ldots\right)$ is the expansion of $n$ with respect to $m$. We often use the following notations. Let $|n|:=\max \left\{k \in \mathbb{N}: n_{k} \neq 0\right\}$ (that is, $\left.M_{|n|} \leq n<M_{|n|+1}\right)$ and $n^{(k)}=\sum_{j=k}^{\infty} n_{j} M_{j}$. Next we introduce on $G_{m}$ an orthonormal system called a Vilenkin-like system.

For $k \in \mathbb{N}$ and $x \in G_{m}$ denote by $r_{k}$ the $k$-th generalized Rademacher function:

$$
r_{k}(x):=\exp \left(2 \pi l \frac{x_{k}}{m_{k}}\right) \quad\left(x \in G_{m}, l:=\sqrt{-1}, k \in \mathbb{N}\right) .
$$

We introduce a so-called Vilenkin-like (or $\psi \alpha$ ) system (see [5], [2]). Let functions

$$
\psi_{n}, \alpha_{n}, \alpha_{j}^{k}: G_{m} \rightarrow \mathbb{C}(n, j, k \in \mathbb{N})
$$

satisfy : 


$$
\begin{gathered}
\alpha_{k}^{j} \text { is measurable with respect to } \mathcal{A}_{k}(j, k \in \mathbb{N}), \\
\left|\alpha_{k}^{j}\right|=\alpha_{k}^{j}(0)=\alpha_{0}^{j}=\alpha_{k}^{0}=1(j, k \in \mathbb{N}), \\
\alpha_{n}:=\prod_{k=0}^{\infty} \alpha_{k}^{n^{(k)}}, \psi_{n}:=\prod_{k=0}^{\infty} r_{k}^{n_{k}}, n^{(k)}:=\sum_{i=k}^{\infty} n_{i} M_{i}(n \in \mathbb{N}) .
\end{gathered}
$$

Let $\chi_{n}:=\psi_{n} \alpha_{n}(n \in \mathbb{N})$. The system $\chi:=\left\{\chi_{n}: n \in \mathbb{N}\right\}$ is called a Vilenkin-like (or $\psi \alpha$ ) system (see [5], [2]). We also introduce the two-variable functions:

$$
\chi_{n}(y, x):=\chi_{n}(y) \bar{\chi}_{n}(x), \quad r_{n}(y, x):=r_{n}(y) \bar{r}_{n}(x) \quad\left(n \in \mathbb{N}, y, x \in G_{m}\right) .
$$

This will not cause misunderstand by clearly making a difference between $\chi_{n}(x)$ and $\chi_{n}(y, x)$. The following lemma is an easy consequence of properties (1.2), (1.3), (1.4). We also need the fact that the generalized Rademacher functions form an orthonormal system (see e.g. $[1,5])$.

Lemma 1 ([5]). Let $t, n, l \in \mathbb{N}, u \in G_{m}$. Then we have that

$$
\int_{I_{t+1}(u)} \chi_{n}(x) \bar{\chi}_{l}(x) d \mu(x) \neq 0
$$

implies $n^{(t+1)}=l^{(t+1)}$.

\section{Example A, the Vilenkin and the Walsh system}

Let $G_{m_{k}}:=Z_{m_{k}}$ be the $m_{k}$-th $\left(2 \leq m_{k} \in \mathbb{N}\right)$ discrete cyclic group $(k \in \mathbb{N})$. That is $Z_{m_{k}}$ can be represented by the set $\left\{0,1, \ldots, m_{k}-1\right\}$, where the group operation is the $\bmod m_{k}$ addition and every subset is open. The group operation on $G_{m}(+)$ is the coordinate-wise addition. $G_{m}$ is called a Vilenkin group. The Vilenkin group for which $m_{k}=2$ for all $k \in \mathbb{N}$ is the Walsh-Paley group. In this case let $\alpha_{k}^{j}(x):=1$, where $j, k \in \mathbb{N}, x \in G_{m}$. The system $\chi:=\left(\chi_{n}: n \in \mathbb{N}\right)$ is the Vilenkin system, where $\chi_{n}:=\prod_{k=0}^{\infty} r_{k}^{n_{k}} \alpha_{k}^{n^{(k)}}=\prod_{k=0}^{\infty} r_{k}^{n_{k}}$. In the case of the Vilenkin group, $m_{k}=2$ for all $k \in \mathbb{N}$, we get the Walsh-Paley system. Properties (1.2), (1.3), (1.4) are trivial fulfilled. For more on Vilenkin and Walsh system and group see e.g. [1] and [6]. For this special case the result of this paper can be found in [8].

\section{Example B, the group of 2-adic ( $m$-adic) integers}

Let $G_{m_{k}}:=\left\{0,1, \ldots, m_{k}-1\right\}$ for all $k \in \mathbb{N}$. Define on $G_{m}$ the following (commutative) addition: Let $x, y \in G_{m}$. Then $x+y=z \in G_{m}$ is defined in a recursive way. $x_{0}+y_{0}=t_{0} m_{0}+z_{0}$, where (of course) $z_{0} \in\left\{0,1, \ldots, m_{0}-1\right\}$ and $t_{0} \in \mathbb{N}$. Suppose that $z_{0}, \ldots, z_{k}$ and $t_{0}, \ldots, t_{k}$ have been defined. Then write $x_{k+1}+y_{k+1}+t_{k}=$ $t_{k+1} m_{k+1}+z_{k+1}$, where $z_{k+1} \in\left\{0,1, \ldots, m_{k+1}-1\right\}$ and $t_{k+1} \in \mathbb{N}$. Then $G_{m}$ is called 
the group of $m$-adic integers (if $m_{k}=2$ for all $k \in \mathbb{N}$, then 2 -adic integers). In this case let

$$
\alpha_{k}^{j}(x):=\left(\exp \left(2 \pi l\left(\frac{x_{k-1}}{m_{k} m_{k-1}}+\cdots+\frac{x_{0}}{m_{k} m_{k-1} \ldots m_{0}}\right)\right)\right)^{j} .
$$

Let $\chi_{n}:=\prod_{k=0}^{\infty} r_{k}^{n_{k}} \alpha_{k}^{n^{(k)}}$. Then the system $\chi:=\left(\chi_{n}: n \in \mathbb{N}\right)$ is the character system of the group of $m$-adic (if $m_{k}=2$ for each $k \in \mathbb{N}$ then 2-adic) integers. Conditions (1.2), (1.3), (1.4) are trivially fulfilled. For more on the group of $m$-adic (if $m_{k}=2$ for each $k \in \mathbb{N}$ then 2-adic) integers see e.g. [2] or [13]. For the case when $m_{k}=2(k \in \mathbb{N})$ the a.e. convergence of the ordinary Marcinkiewicz means were discussed by Blahota and Gát in [2]. That is, the results of this paper are new on the two-dimensional group of $m$-adic integers. Not only with respect to the general case $\alpha: \mathbb{N}^{2} \rightarrow \mathbb{N}^{2}$ but also for $\alpha_{1}(n)=\alpha_{2}(n)=n$. Besides, the same can be said in the situation of the Example C below.

\section{Example $\mathrm{C}$, a system in the field of number theory}

Let

$$
\chi_{n}(x):=\exp \left(2 \pi \imath \sum_{j=0}^{\infty} \frac{n_{j}}{M_{j+1}} \sum_{i=0}^{\infty} x_{i} M_{i}\right)
$$

for $n \in \mathbb{N}$ and $x \in G_{m}$. Then

$$
\chi_{n}(x)=\exp \left(2 \pi l\left(\sum_{k=0}^{\infty} \frac{n_{k} x_{k}}{m_{k}}+\sum_{k=0}^{\infty} \frac{n_{k}}{M_{k+1}} \sum_{i=0}^{k-1} x_{i} M_{i}\right)\right)=\psi_{n}(x) \alpha_{n}(x),
$$

where $\alpha_{k}^{n^{(k)}}(x)=\exp \left(2 \pi l \frac{n_{k}}{M_{k+1}} \sum_{i=0}^{k-1} x_{i} M_{i}\right)$. Then, $\chi:=\left(\chi_{n}: n \in \mathbb{N}\right)$ is a Vilenkinlike system (introduced in [5]) which is a useful tool in the approximation theory of limit periodic, almost even arithmetical functions [5] and [4]. Again, properties (1.2), (1.3), (1.4) are trivially fulfilled. This system (on Vilenkin groups) was a new tool in order to investigate limit periodic arithmetical functions. For the definition of these arithmetical functions see also the book of Mauclaire [15].

Define the Fourier coefficients, the partial sums of the Fourier series and the Dirichlet kernels with respect to the Vilenkin-like system $\chi$ as follows

$$
\begin{aligned}
& \hat{f}(n):=\int_{G_{m}} f \bar{\chi}_{n} d \mu, \quad S_{n} f:=\sum_{k=0}^{n-1} \hat{f}(k) \chi_{k}, \\
& D_{n}(y, x):=\sum_{k=0}^{n-1} \chi_{k}(y) \bar{\chi}_{k}(x)=\sum_{k=0}^{n-1} \chi_{k}(y, x) \quad\left(n \in \mathbb{N}, \quad y, x \in G_{m}, f \in L^{1}\left(G_{m}\right)\right) .
\end{aligned}
$$

It is well-known that

$$
S_{n} f(y)=\int_{G_{m}} f(x) D_{n}(y, x) d \mu(x), \quad\left(n \in \mathbb{N}, y \in G_{m}, f \in L^{1}\left(G_{m}\right)\right) .
$$


It is also well-known [5] that

$$
\begin{aligned}
& D_{M_{n}}(y, x)= \begin{cases}M_{n}, & \text { if } y \in I_{n}(x) \\
0, & \text { if } y \notin I_{n}(x)\end{cases} \\
& D_{n}(y, x)=\chi_{n}(y) \bar{\chi}_{n}(x) \sum_{j=0}^{\infty} D_{M_{j}}(y, x) \sum_{p=m_{j}-n_{j}}^{m_{j}-1} r_{j}^{p}(y) \bar{r}_{j}^{p}(x), \\
& S_{M_{n}} f(x)=M_{n} \int_{I_{n}(x)} f d \mu=E_{n} f(x) \quad\left(f \in L^{1}\left(G_{m}\right), n \in \mathbb{N}\right), \\
& D_{n}(y, x)=\chi_{n}(y) \bar{\chi}_{n}(x)\left(\sum_{j=0}^{t-1} n_{j} M_{j}+M_{t} \sum_{i=m_{t}-n_{t}}^{m_{t}-1} r_{t}^{i}(y) \bar{r}_{t}^{i}(x)\right), \\
& y \in I_{t}(x) \backslash I_{t+1}(x), t \in \mathbb{N} .
\end{aligned}
$$

Next, we introduce some notation with respect to the theory of two-dimensional Vilenkin-like systems. Let $\tilde{m}$ be a sequence like $m$. The relation between the sequence $\left(\tilde{m}_{n}\right)$ and $\left(\tilde{M}_{n}\right)$ is the same as between sequence $\left(m_{n}\right)$ and $\left(M_{n}\right)$. The group $G_{m} \times G_{\tilde{m}}$ is called a two-dimensional Vilenkin group. The normalized Haar measure is denoted by $\mu$, just as in the one-dimensional case. It will not cause any misunderstood. In this paper we also suppose that $m=\tilde{m}$. The two-dimensional Fourier coefficients, the rectangular partial sums of the Fourier series, the Dirichlet kernels, the Marcinkiewicz means, and the Marcinkiewicz kernels with respect to the twodimensional Vilenkin-like system are defined as follows:

$$
\begin{aligned}
& \hat{f}\left(n_{1}, n_{2}\right):=\int_{G_{m} \times G_{\tilde{m}}} f\left(x^{1}, x^{2}\right) \bar{\chi}_{n_{1}}\left(x^{1}\right) \bar{\chi}_{n_{2}}\left(x^{2}\right) d \mu\left(x^{1}, x^{2}\right), \\
& S_{n_{1}, n_{2}} f\left(y^{1}, y^{2}\right):=\sum_{k_{1}=0}^{n_{1}-1} \sum_{k_{2}=0}^{n_{2}-1} \hat{f}\left(k_{1}, k_{2}\right) \chi_{k_{1}}\left(y^{1}\right) \chi_{k_{2}}\left(y^{2}\right), \\
& D_{n_{1}, n_{2}}(y, x)=D_{n_{1}}\left(y^{1}, x^{1}\right) D_{n_{2}}\left(y^{2}, x^{2}\right) \\
& :=\sum_{k_{1}=0}^{n_{1}-1} \sum_{k_{2}=0}^{n_{2}-1} \chi_{k_{1}}\left(y^{1}\right) \chi_{k_{2}}\left(y^{2}\right) \bar{\chi}_{k_{1}}\left(x^{1}\right) \bar{\chi}_{k_{2}}\left(x^{2}\right), \\
& \sigma_{n} f:=\frac{1}{n} \sum_{j=0}^{n-1} S_{j, j} f, \\
& K_{n}(y, x):=\frac{1}{n} \sum_{j=0}^{n-1} D_{j, j}(y, x), \\
& \left(y=\left(y^{1}, y^{2}\right), x=\left(x^{1}, x^{2}\right) \in G_{m} \times G_{m}, n \in \mathbb{N}\right) .
\end{aligned}
$$


It is also well-known that

$$
\sigma_{n} f(y)=\int_{G_{m} \times G_{m}} f(x) K_{n}(y, x) d \mu(x)=: f * K_{n}(y) .
$$

\section{RESUlt}

Now, turn our attention to the generalization of Marcienkiewicz means with respect to two dimensional bounded Vilenkin-like systems. The two-dimensional generalized Marcinkiewicz kernels and Marcinkiewicz means, with respect to the twodimensional Vilenkin-like system are defined as follows: Let $\alpha=\left(\alpha_{1}, \alpha_{2}\right): \mathbb{N}^{2} \rightarrow \mathbb{N}^{2}$ be a function. (From now functions $\alpha_{1}, \alpha_{2}$ play the role of indices. We know that in the first part of the article the function $\alpha_{n}$ appeared in the definition of the Vilenkinlike (or $\psi \alpha$ system), but this will not cause any misunderstanding.) Define the following generalized Marcinkiewicz kernels and means respectively:

$$
\begin{aligned}
& M_{n}^{\alpha}(y, x):=\frac{1}{n} \sum_{k=0}^{n-1} D_{\alpha_{1}(|n|, k)}\left(y^{1}, x^{1}\right) D_{\alpha_{2}(|n|, k)}\left(y^{2}, x^{2}\right), \\
& t_{n}^{\alpha} f:=f * M_{n}^{\alpha} \quad\left(f \in L^{1}\left(G_{m}^{2}\right), n \in \mathbb{P}\right) .
\end{aligned}
$$

This concept of Marcinkiewicz-like kernels and means is due to Gát [7].

The main aim of this paper is to give a class of functions $\alpha$ for we have the a.e. convergence relation $t_{n}^{\alpha} f \rightarrow f$ for each integrable two variable function with respect to two dimensional bounded Vilenkin-like systems. To investigate this the following properties play a prominent role $(\mathrm{x} 0 \operatorname{Car}(B)$ denotes cardinality of the set $B)$,

$$
\begin{gathered}
\operatorname{Car}\left\{l \in \mathbb{N}: \alpha_{j}(|n|, l)=\alpha_{j}(|n|, k), l<n\right\} \leq C \quad(k<n, n \in \mathbb{P}, j=1,2), \\
\max \left\{\alpha_{j}(|n|, k): k<n\right\} \leq C n \quad(n \in \mathbb{P}, j=1,2) .
\end{gathered}
$$

We emphasize that the function $\alpha$ has the properties (2.1) and (2.2) everywhere in this paper. Our first aim is to prove that the operator $t_{*}^{\alpha}:=\sup _{n \in \mathbb{P}}\left|t_{n}^{\alpha}\right|$ is of weak type $\left(L^{1}, L^{1}\right)$. In order to do this we need a sequence of lemmas. The second lemma is the base of the proof of Theorems 1 and 2. The Walsh-Paley version of Theorems 1 and 2 are due to Gát [7]. That is, we generalize a result of the first author. Moreover, techniques of papers [7] and [8] will also be used in the proof of the forthcoming lemmas.

Denote for $k \in \mathbb{N}, x \in G_{m}, J_{k}(x)=I_{k}(x) \backslash I_{k+1}(x)$ and recall also that

$$
n^{(s)}=\sum_{k=s}^{\infty} n_{k} M_{k}, \quad n^{(0)}=n, \quad n^{(|n|+1)}=0 \quad(n, s \in \mathbb{N}) .
$$

Besides, for $x, y \in G_{m}^{2}, A, n, s, j, k \in \mathbb{N}$ let

$$
\Phi\left(A, n^{(s+1)}+j M_{s}+k, y, x\right)=D_{\alpha_{1}\left(A, n^{(s+1)}+j M_{s}+k\right)}\left(y^{1}, x^{1}\right) D_{\alpha_{2}\left(A, n^{(s+1)}+j M_{s}+k\right)}\left(y^{2}, x^{2}\right) .
$$


Lemma 2. Let $t^{1}, t^{2}, A, s \in \mathbb{N}, s \leq A$ and $y \in G_{m}^{2}$. Then,

$$
\int_{J_{t^{1}}\left(y^{1}\right) \times J_{t^{2}}\left(y^{2}\right)} \sup _{|n|=A}\left|\sum_{j=0}^{n_{s}-1} \sum_{k=0}^{M_{s}-1} \Phi\left(A, n^{(s+1)}+j M_{s}+k, y, x\right)\right| d \mu(x) \leq C\left(M_{A} M_{t^{1}}\right)^{\frac{1}{2}} .
$$

Proof. For fixed $t=\left(t^{1}, t^{2}\right), s, A$ we discuss the integral

$$
\int_{J_{t^{1}}\left(y^{1}\right) \times J_{t^{2}}\left(y^{2}\right)} \sup _{|n|=A}\left|\sum_{j=0}^{n_{s}-1} \sum_{k=0}^{M_{s}-1} \Phi\left(A, n^{(s+1)}+j M_{s}+k, y, x\right)\right| d \mu(x) .
$$

Check the function $\sum_{j=0}^{n_{s}-1} \sum_{k=0}^{M_{s}-1} \Phi\left(A, n^{(s+1)}+j M_{s}+k, y, x\right)$ on the set $J_{t^{1}}\left(y^{1}\right) \times J_{t^{2}}\left(y^{2}\right)$. Since we have $x^{2} \in J_{t^{2}}\left(y^{2}\right)$, then by (1.5) we have $\left|D_{j}\left(y^{2}, x^{2}\right)\right| \leq C M_{t^{2}}$ for each $j \in \mathbb{N}$ (this inequality is due to the fact that the Vilenkin space is bounded) and consequently by (1.5) $\left|D_{\alpha_{2}\left(A, n^{(s+1)}+j M_{s}+k\right)}\left(y^{2}, x^{2}\right)\right| \leq C M_{t^{2}}$.

On the other hand, again by (1.5) for $x^{1} \in J_{t^{1}}\left(y^{1}\right)$ we have

$$
\begin{aligned}
& D_{\alpha_{1}\left(A, n^{(s+1)}+j M_{s}+k\right)}\left(y^{1}, x^{1}\right)=\chi_{\left[\alpha_{1}\left(A, n^{(s+1)}+j M_{s}+k\right)\right]^{\left(t^{1}\right)}}\left(y^{1}\right) \\
& \bar{\chi}_{\left[\alpha_{1}\left(A, n^{(s+1)}+j M_{s}+k\right)\right]^{\left(t^{1}\right)}}\left(x^{1}\right) \times\left[\sum_{w=0}^{t^{1}-1}\left[\alpha_{1}\left(A, n^{(s+1)}+j M_{s}+k\right)\right]_{w} M_{w}\right. \\
& \left.+\sum_{i=m_{t^{1}}-\left(\alpha_{1}\left(A, n^{(s+1)}+j M_{s}+k\right)\right)_{t^{1}}}^{m_{t^{1}}-1} r_{t^{1}}^{i}\left(y^{1}\right) \bar{r}_{t^{1}}^{i}\left(x^{1}\right) M_{t^{1}}\right] .
\end{aligned}
$$

The function

$$
\sum_{w=0}^{t^{1}-1}\left[\alpha_{1}\left(A, n^{(s+1)}+j M_{s}+k\right)\right]_{w} M_{w}+\sum_{i=m_{t^{1}}-\left(\alpha_{1}\left(A, n^{(s+1)}+j M_{s}+k\right)\right)_{t^{1}}}^{m_{t^{1}}-1} r_{t^{1}}^{i}\left(y^{1}\right) \bar{r}_{t^{1}}^{i}\left(x^{1}\right) M_{t^{1}}
$$

depends only on $x_{t^{1}}^{1}$ as $x$ varies (and not on the other coordinates of $x^{1}$ ), so its absolute value is bounded by $C M_{t}$. Besides,

$$
\begin{aligned}
& \left|\sum_{j=0}^{n_{s}-1} \sum_{k=0}^{M_{s}-1} D_{\alpha_{1}\left(A, n^{(s+1)}+j M_{s}+k\right)}\left(y^{1}, x^{1}\right) D_{\alpha_{2}\left(A, n^{(s+1)}+j M_{s}+k\right)}\left(y^{2}, x^{2}\right)\right|^{2} \\
& =\sum_{j, h=0}^{n_{s}-1} \sum_{k, l=0}^{M_{s}-1} D_{\alpha_{1}\left(A, n^{(s+1)}+j M_{s}+k\right)}\left(y^{1}, x^{1}\right) \bar{D}_{\alpha_{1}\left(A, n n^{(s+1)}+h M_{s}+l\right)}\left(y^{1}, x^{1}\right) \\
& \times D_{\alpha_{2}\left(A, n^{(s+1)}+j M_{s}+k\right)}\left(y^{2}, x^{2}\right) \bar{D}_{\alpha_{2}\left(A, n^{(s+1)}+h M_{s}+l\right)}\left(y^{2}, x^{2}\right) .
\end{aligned}
$$

Therefore, apply the Cauchy-Bunyakovsky-Schwarz inequality: 


$$
\begin{aligned}
& \int_{J_{t^{2}}\left(y^{2}\right)}\left[\int_{J_{t^{1}}\left(y^{1}\right)} \sup _{|n|=A}\left|\sum_{j=0}^{n_{s}-1} \sum_{k=0}^{M_{s}-1} \Phi\left(A, n^{(s+1)}+j M_{s}+k, y^{1}, y^{2}, x^{1}, x^{2}\right)\right| d \mu\left(x^{1}\right)\right] d \mu\left(x^{2}\right) \\
& \leq \int_{J_{t^{2}}\left(y^{2}\right)^{t^{1}}} M^{\frac{-1}{2}}\left[\int_{J_{t_{1}}\left(y^{1}\right)} \sup _{|n|=A} \mid \sum_{j=0}^{n_{s}-1} \sum_{k=0}^{M_{s}-1} \Phi\left(A, n^{(s+1)}+j M_{s}\right.\right. \\
& \left.\left.+k, y^{1}, y^{2}, x^{1}, x^{2}\right)\left.\right|^{2} d \mu\left(x^{1}\right)\right]^{\frac{1}{2}} d \mu\left(x^{2}\right) \\
& \leq \int_{J^{2}\left(y^{2}\right)} M_{t^{1}}^{\frac{-1}{2}}\left[\int_{t_{t_{1}}\left(y^{1}\right)} \sup _{|n|=A} \sum_{j, h=0}^{n_{s}-1} \sum_{k, l=0}^{M_{s}-1} D_{\alpha_{1}\left(A, n^{(s+1)}+j M_{s}+k\right)}\left(y^{1}, x^{1}\right) \bar{D}_{\alpha_{1}\left(A, n^{(s+1)}+h M_{s}+l\right)}\left(y^{1}, x^{1}\right)\right. \\
& \left.\times D_{\alpha_{2}\left(A, n^{(s+1)}+j M_{s}+k\right)}\left(y^{2}, x^{2}\right) \bar{D}_{\alpha_{2}\left(A, n^{(s+1)}+h M_{s}+l\right)}\left(y^{2}, x^{2}\right) d \mu\left(x^{1}\right)\right] d \mu\left(x^{2}\right) \\
& =\int_{J_{t^{2}}\left(y^{2}\right)} M_{t^{1}}^{\frac{-1}{2}}\left[\int_{J_{t^{1}}\left(y^{1}\right)} \sup _{|n|=A} \sum_{j, h=0}^{n_{s}-1} \sum_{k, l=0}^{M_{s}-1} \chi_{\left[\alpha_{1}\left(A, n^{(s+1)}+j M_{s}+k\right)\right.}\right]^{\left(t^{1}\right)}\left(y^{1}, x^{1}\right) \\
& \bar{\chi}_{\left[\alpha_{1}\left(A, n^{(s+1)}+h M_{s}+l\right)\right]^{\left(t^{1}\right)}}\left(y^{1}, x^{1}\right) \\
& \times\left(\sum_{w=0}^{t^{1}-1} \alpha_{1}\left(A, n^{(s+1)}+j M_{s}+k\right)_{w} M_{w}+\sum_{a=m_{t^{1}}-\left[\alpha_{1}\left(A, n^{(s+1)}\right)+j M_{s}+k\right]_{t^{1}}}^{m_{t^{1}}-1} r_{t^{1}}^{a}\left(y^{1}, x^{1}\right)\right) \\
& \times\left(\sum_{v=0}^{t^{1}-1} \alpha_{1}\left(A, n^{(s+1)}+h M_{s}+l\right)_{v} M_{v}+\sum_{b=m_{t^{1}}-\left[\alpha_{1}\left(A, n^{(s+1)}\right)+h M_{s}+l\right]_{t^{1}}}^{m_{t^{1}}-1} \overline{t^{b}}\left(y^{1}, x^{1}\right)\right) \\
& \left.D_{\alpha_{2}\left(A, n^{(s+1)}+j M_{s}+k\right)}\left(x^{2}\right) \bar{D}_{\alpha_{2}\left(A, n^{(s+1)}+h M_{s}+l\right)}\left(y^{2}, x^{2}\right) d \mu\left(x^{1}\right)\right]^{\frac{1}{2}} d \mu\left(x^{2}\right)=: B^{1} .
\end{aligned}
$$

Since $n^{(s+1)}$ depends only on natural numbers $n_{s+1}, \ldots, n_{A-1}, n_{A}$, then the supreme $\sup _{n \in \mathbb{N}:|n|=A}$ above also depends only on $n_{s}, n_{s+1}, \ldots, n_{A-1}, n_{A}$. Thus, by $|n|=A$

$$
\begin{gathered}
B^{1} \leq \int_{J_{t^{2}}\left(y^{2}\right)} M_{t^{1}}^{-\frac{1}{2}}\left[\sum_{n_{A}=1}^{m_{A}-1} \sum_{n_{A-1}=0}^{m_{A-1}-1} \cdots \sum_{n_{s+1}=0}^{m_{s+1}-1} \sum_{n_{s}=0}^{m_{s}-1} \int_{J^{1}} \sum_{\left(y^{1}\right)} \sum_{j, h=0}^{n_{s}-1} \sum_{k, l=0}^{M_{s}-1}\right. \\
\chi_{\left[\alpha_{1}\left(A, n^{(s+1)}+j M_{s}+k\right)\right]^{\left(t^{1}\right)}\left(y^{1}, x^{1}\right) \bar{\chi}_{\left[\alpha_{1}\left(A, n^{(s+1)}+h M_{s}+l\right)\right]^{\left(t^{1}\right)}\left(y^{1}, x^{1}\right)}} \times\left(\sum_{w=0}^{t^{1}-1} \alpha_{1}\left(A, n^{(s+1)}+j M_{s}+k\right)_{w} M_{w}+\sum_{a=m_{t^{1}}-\left[\alpha_{1}\left(A, n^{(s+1)}\right)+j M_{s}+k\right]_{t}{ }^{1}}^{m_{t^{1}}-1} r^{a}\left(y^{1}, x^{1}\right)\right)
\end{gathered}
$$




$$
\begin{aligned}
& \times\left(\sum_{v=0}^{t^{1}-1} \alpha_{1}\left(A, n^{(s+1)}+h M_{s}+l\right)_{v} M_{v}+\sum_{b=m_{t^{1}}-\left[\alpha_{1}\left(A, n^{(s+1)}\right)+h M_{s}+l\right]_{t^{1}}}^{m_{t^{1}}-1} \overline{r^{b}}\left(y^{1}, x^{1}\right)\right) \\
& \left.\times D_{\alpha_{2}\left(A, n^{(s+1)}+j M_{s}+k\right)}\left(y^{1}, x^{1}\right) \bar{D}_{\alpha_{2}\left(A, n^{(s+1)}+h M_{s}+l\right)}\left(y^{2}, x^{2}\right) d \mu\left(x^{1}\right)\right]^{\frac{1}{2}} d \mu\left(x^{2}\right) \\
& =\int_{t^{2}\left(y^{2}\right)} M_{t^{1}}^{-\frac{1}{2}}\left[\sum_{n_{A}=1}^{m_{A}-1} \sum_{n_{A-1}=0}^{m_{A-1}} \cdots \sum_{n_{s+1}=0}^{m_{s+1}-1} \sum_{n_{s}=0}^{m_{s}-1} \sum_{j, h=0}^{n_{s}-1} \sum_{k, l=0}^{M_{s}-1}\right. \\
& \int_{J_{t^{1}}\left(y^{1}\right)}\left(\sum_{w=0}^{t^{1}-1} \alpha_{1}\left(A, n^{(s+1)}+j M_{S}+k\right)_{w} M_{w}+\sum_{a=m_{t^{1}}-\left[\alpha_{1}\left(A, n^{(s+1)}\right)+j M_{s}+k\right]_{t^{1}}}^{m_{t^{1}}-1} r_{t_{1}}^{a}\left(y^{1}, x^{1}\right)\right) \\
& \times\left(\sum_{v=0}^{t^{1}-1} \alpha_{1}\left(A, n^{(s+1)}+h M_{s}+l\right)_{v} M_{v}+\sum_{b=m_{t^{1}}-\left[\alpha_{1}\left(A, n^{(s+1)}\right)+h M_{s}+l\right]_{t^{1}}}^{m_{t^{1}}-1} \overline{r^{b}}\left(y^{1}, x^{1}\right)\right) \\
& \times \chi_{\left[\alpha_{1}\left(A, n^{(s+1)}+j M_{s}+k\right)\right]^{\left(t^{1}\right)}}\left(y^{1}, x^{1}\right) \bar{\chi}_{\left[\alpha_{1}\left(A, n^{(s+1)}+h M_{s}+l\right)\right]^{\left(t^{1}\right)}}\left(y^{1}, x^{1}\right) d \mu\left(x^{1}\right) \\
& \left.\times D_{\alpha_{2}\left(A, n^{(s+1)}+j M_{s}+k\right)}\left(y^{2}, x^{2}\right) \bar{D}_{\alpha_{2}\left(A, n^{(s+1)}+h M_{s}+l\right)}\left(y^{2}, x^{2}\right)\right]^{\frac{1}{2}} d \mu\left(x^{2}\right) \\
& =\int_{J^{2}\left(y^{2}\right)} M_{t^{1}}^{-\frac{1}{2}}\left[\sum_{n_{A}=1}^{m_{A}-1} \sum_{n_{A-1}=0}^{m_{A}-1} \cdots \sum_{n_{s+1}=0}^{m_{s+1}-1} \sum_{n_{s}=0}^{m_{s}-1} \sum_{j, h=0}^{n_{s}-1} \sum_{k, l=0}^{M_{s}-1}\right. \\
& \left(\sum_{w=0}^{t^{1}-1} \sum_{v=0}^{t^{1}-1} \alpha_{1}\left(A, n^{(s+1)}+j M_{s}+k\right)_{w} M_{w} \alpha_{1}\left(A, n^{(s+1)}+h M_{s}+l\right)_{v} M_{v}\right. \\
& \times \int_{J_{t^{1}}\left(y^{1}\right)} \chi_{\left[\alpha_{1}\left(A, n^{(s+1)}+j M_{s}+k\right)\right]^{\left(t^{1}\right)}}\left(y^{1}, x^{1}\right) \bar{\chi}_{\left[\alpha_{1}\left(A, n^{(s+1)}+h M_{s}+l\right)\right]^{\left(t^{1}\right)}}\left(y^{1}, x^{1}\right) d \mu\left(x^{1}\right) \\
& +\sum_{w=0}^{t^{1}-1} \sum_{b=m_{t^{1}}-\left[\alpha_{1}\left(A, n^{(s+1)}\right)+h M_{s}+l\right]_{t^{1}}}^{m_{t^{1}}-1} \alpha_{1}\left(A, n^{(s+1)}+j M_{s}+k\right)_{w} M_{w} \\
& \left.\times \int_{J_{t^{1}}\left(y^{1}\right)} \chi_{\left[\alpha_{1}\left(A, n^{(s+1)}+j M_{s}+k\right)\right]^{\left(t^{1}\right)}}\left(x^{1}\right) \bar{\chi}_{\left[\alpha_{1}\left(A, n^{(s+1)}+h M_{s}+l\right)\right]}\right]^{\left(t^{1}\right)}\left(x^{1}\right) \bar{r}_{t^{1}}^{b}\left(y^{1}, x^{1}\right) d \mu\left(x^{1}\right) \\
& +\sum_{a=m_{t^{1}}-\left[\alpha_{1}\left(A, n^{(s+1)}\right)+j M_{s}+k\right]_{t^{1}}}^{m_{t^{1}}-1} \sum_{v=0}^{t^{1}-1} \alpha_{1}\left(A, n^{(s+1)}+h M_{s}+l\right)_{v} M_{v} \\
& \times \int_{J_{t^{1}}\left(y^{1}\right)} \chi_{\left[\alpha_{1}\left(A, n^{(s+1)}+j M_{s}+k\right)\right]^{\left(t^{1}\right)}}\left(y^{1}, x^{1}\right) \bar{\chi}_{\left[\alpha_{1}\left(A, n^{(s+1)}+h M_{s}+l\right)\right]^{\left(t^{1}\right)}}\left(x^{1}\right) r_{t_{1}}^{a}\left(y^{1}, x^{1}\right) d \mu\left(x^{1}\right)
\end{aligned}
$$




$$
\begin{gathered}
+\sum_{a=m_{t^{1}}-\left[\alpha_{1}\left(A, n^{(s+1)}\right)+j M_{s}+k\right]_{t^{1}} 1}^{m_{t^{1}}-1} \sum_{b=m_{t^{1}}-\left[\alpha_{1}\left(A, n^{(s+1)}\right)+h M_{s}+l\right]_{t^{1}}}^{m_{t^{1}}-1} \chi_{J_{t^{1}}\left(y^{1}\right)} \chi_{\left[\alpha_{1}\left(A, n^{(s+1)}+j M_{s}+k\right)\right]^{\left(t^{1}\right)}}\left(y^{1}, x^{1}\right) \bar{\chi}_{\left[\alpha_{1}\left(A, n^{(s+1)}+h M_{s}+l\right)\right]^{\left(t^{1}\right)}}\left(y^{1}, x^{1}\right) \\
\left.r_{t_{1}}^{a}\left(y^{1}, x^{1}\right) \bar{r}_{t^{1}}^{b}\left(y^{1}, x^{1}\right) d \mu\left(x^{1}\right)\right) \times D_{\alpha_{2}\left(A, n^{(s+1)}+j M_{s}+k\right)}\left(y^{2}, x^{2}\right) \\
\left.\bar{D}_{\alpha_{2}\left(A, n^{(s+1)}+h M_{s}+l\right)}\left(y^{2}, x^{2}\right)\right]^{\frac{1}{2}} d \mu\left(x^{2}\right)=: \sum_{q=1}^{4} B^{2, q}=: B^{2} .
\end{gathered}
$$

Before we estimate $B^{2}$, (that is, any of $B^{2, q}$ ) let us determine the possible number of $k, l^{\prime} s$ such that the integral

$$
\begin{aligned}
& \int_{J_{t^{1}}\left(y^{1}\right)} \chi_{\left[\alpha_{1}\left(A, n^{(s+1)}+j M_{s}+k\right)\right]^{\left(t^{1}\right)}}\left(y^{1}, x^{1}\right) \bar{\chi}_{\left[\alpha_{1}\left(A, n^{(s+1)}+h M_{s}+l\right)\right]^{\left(t^{1}\right)}}\left(y^{1}, x^{1}\right) \\
& \times r_{t_{1}}^{a}\left(y^{1}, x^{1}\right) \overline{r_{t^{1}}^{b}}\left(y^{1}, x^{1}\right) d \mu\left(x^{1}\right) \neq 0 .
\end{aligned}
$$

(Recall that in the case of $B^{2,1}$ we have $a=b=0$, in the case of $B^{2,2}$ we have $a=0$ and in the case of $B^{2,3}$ we have $b=0$.) Suppose the integral,

$$
\begin{aligned}
& \int_{J_{t^{1}}\left(y^{1}\right)} \chi_{\left[\alpha_{1}\left(A, n^{(s+1)}+j M_{s}+k\right)\right]^{\left(t^{1}\right)}}\left(y^{1}, x^{1}\right) \bar{\chi}_{\left[\alpha_{1}\left(A, n^{(s+1)}+h M_{s}+l\right)\right]^{\left(t^{1}\right)}}\left(y^{1}, x^{1}\right) \\
& \times r_{t_{1}}^{a}\left(y^{1}, x^{1}\right) \overline{r^{b}}\left(y^{1}, x^{1}\right) d \mu\left(x^{1}\right) \neq 0
\end{aligned}
$$

for some $a, b \in\left\{0,1, \ldots, m_{t_{1}}-1\right\}$. Then use Lemma 1 and (1.2)-(1.4). Consequently, the $\left(t^{1}+1\right)^{t h},\left(t^{1}+2\right)^{t h}, \ldots$, coordinates of $\alpha_{1}\left(A, n^{(s+1)}+j M_{s}+k\right)$ and $\alpha_{1}\left(A, n^{(s+1)}+\right.$ $\left.h M_{s}+l\right)$ should be equal. The absolute value of this integral is not greater than $1 / M_{t^{1}}$. Since by (2.1) we have that for every $k$, there exist a bounded number of $l^{\prime} s$ for which $\alpha_{1}\left(A, n^{(s+1)}+j M_{s}+k\right)=\alpha_{1}\left(A, n^{(s+1)}+h M_{s}+l\right)$. This gives that for every $k$, there exist at most $C M_{t^{1}}$ number of $l^{\prime} s$ for which this integral is not zero. Consequently, by

$$
\sum_{w=0}^{t^{1}-1}\left[\alpha_{1}\left(A, n^{(s+1)}+j M_{s}+k\right)\right]_{w} M_{w} \leq C M_{t^{1}}, \quad\left|D_{\alpha_{2}\left(A, n^{(s+1)}+j M_{s}+k\right)}\left(y^{2}, x^{2}\right)\right| \leq C M_{t^{2}}
$$

we have

$$
B^{2,1} \leq C \int_{J^{2}\left(y^{2}\right)} M_{t^{1}}^{-\frac{1}{2}}\left[\sum_{n_{A}=1}^{m_{A}-1} \sum_{n_{A-1}=0}^{m_{A-1}} \cdots \sum_{n_{s+1}=0}^{m_{s+1}-1} \sum_{n_{s}=0}^{m_{s}-1} \sum_{j, h=0}^{n_{s}-1} \sum_{k=0}^{M_{s}-1} M_{t^{1}} M_{t^{1}}^{2} \frac{1}{M_{t^{1}}} M_{t^{2}}^{2}\right]^{\frac{1}{2}} d \mu\left(x^{2}\right) .
$$

(Keep in mind that the Vilenkin space is bounded. That is, $m_{j} \leq C$ for all $j \in \mathbb{N}$.) By the very same steps we get the identical upper bound also for $B^{2,2}, B^{2,3}$ and $B^{2,4}$. Then, also for their sum. That is, 


$$
\begin{aligned}
& B^{2} \leq C \int_{J^{2}\left(y^{2}\right)} M_{t^{1}}^{-\frac{1}{2}}\left[\sum_{n_{A}=1}^{m_{A}-1} \sum_{n_{A-1}=0}^{m_{A-1}-1} \cdots \sum_{n_{s+1}=0}^{m_{s+1}-1} \sum_{n_{s}=0}^{m_{s}-1}\left(\left(M_{t^{1}} M_{t^{2}}\right)^{2} M_{S} M_{t^{1}} M_{t^{1}}^{-1}\right)\right]^{\frac{1}{2}} d \mu \\
& \leq C M_{t^{2}}^{-1} M_{t^{1}}^{\frac{-1}{2}}\left[\frac{M_{A}}{M_{S}} M_{t^{1}}^{2} M_{t^{2}}^{2} M_{s}\right]^{\frac{1}{2}} \leq C\left(M_{A} M_{t^{1}}\right)^{\frac{1}{2}} .
\end{aligned}
$$

This means,

$$
\begin{aligned}
& \int_{J_{t^{1}}\left(y^{1}\right) \times J_{t^{2}}\left(y^{2}\right)} \sup _{|n|=A}\left|\sum_{j=0}^{n_{s}-1} \sum_{k=0}^{M_{s}-1} \Phi\left(A, n^{(s+1)}+j M_{s}+k, y, x\right)\right| d \mu(x) \\
& \leq C\left(M_{A} M_{t^{1}}\right)^{\frac{1}{2}} .
\end{aligned}
$$

This completes the proof of Lemma 2.

Lemma 3. Let $a \in \mathbb{N}, y \in G_{m}^{2}$. Then,

$$
\sum_{t^{1}=0 t^{2}=t^{1}}^{a-1} \int_{J^{1}}^{\infty} \int_{\left.y^{1}\right) \times J_{t^{2}}\left(y^{2}\right)} \sup _{A \geq a|n|=A} \frac{1}{M_{A}} \sum_{s=t^{1}}^{A} \sum_{j=0}^{n_{s}-1}\left|\sum_{k=0}^{M_{s}-1} \Phi\left(A, n^{(s+1)}+j M_{s}+k, y, x\right)\right| d \mu(x) \leq C .
$$

Proof. The inequality in Lemma 2 immediately gives the following result:

$$
\begin{gathered}
\left.\sum_{t^{1}=0}^{a-1} \sum_{t^{2}=t^{1}}^{\infty} \int_{J^{1}}\left(y^{1}\right) \times J_{t^{2}} y^{2}\right) \\
\mid \sum_{A=\max \left(a, t^{2}-c\right)} \sup _{|n|=A} \frac{1}{M_{A}} \sum_{s=t^{1}}^{A} \sum_{j=0}^{n_{s}-1} \\
\leq C \sum_{t^{1}=0}^{a-1} \sum_{t^{2}=t^{1}}^{\infty} \sum_{A=\max \left(a, t^{2}-c\right)}^{\infty} \sum_{s=t^{1}}^{A}\left(M_{t^{1}} M_{A}^{-1}\right)^{\frac{1}{2}} \\
=C \sum_{t^{1}=0 t^{2}=t^{1}}^{a-1} \sum_{A=\max \left(a, t^{2}-c\right)}^{\infty}\left(A-t^{1}+1\right)\left(M_{t^{1}} M_{A}^{-1}\right)^{\frac{1}{2}} \\
\leq C \sum_{t^{1}=0}^{a-1} \sum_{t^{2}=t^{1}}^{\infty}\left(\max \left(a, t^{2}\right)-t^{1}\right)\left(M_{t^{1}} M_{\max \left(a, t^{2}-c\right)}^{-1}\right)^{\frac{1}{2}} \\
\leq C \sum_{t^{1}=0}^{a-1} \sum_{t^{2}=t^{1}}^{\infty}\left(\max \left(a, t^{2}\right)-t^{1}\right)\left(M_{t^{1}} M_{\max \left(a, t^{2}\right)}^{-1}\right)^{\frac{1}{2}} \\
\leq C \sum_{t^{1}=0}^{a-1} \sum_{t^{2}=t^{1}}^{a-1}\left(a-t^{1}\right)\left(M_{t^{1}} M_{a}^{-1}\right)^{\frac{1}{2}}+C \sum_{t^{1}=0}^{a-1} \sum_{t^{2}=a+1}^{\infty}\left(t^{2}-t^{1}\right)\left(M_{t^{1}} M_{t^{2}}^{-1}\right)^{\frac{1}{2}} \leq C .
\end{gathered}
$$


This estimation used the fact that $M_{\max \left(a, t^{2}\right)} \leq C M_{\max \left(a, t^{2}-c\right)}$ which from the boundedness of the system (i.e. the Vilenkin space). This inequality shows that if we want to complete the proof of this lemma, then we have to discuss also the case when $\sup _{t^{2}-c>A \geq a}$. This follows that $t^{2}$ should be at least $a+c$. That is, we have to prove that the following integral is bounded

$$
\begin{aligned}
& \sum_{t^{1}=0}^{a-1} \sum_{t^{2}=a+c}^{\infty} \int_{J^{1}\left(y^{1}\right) \times J_{t^{2}}\left(y^{2}\right)} \sup _{t^{2}-c>A \geq a} \sup _{|n|=A} \frac{1}{M_{A}} \sum_{s=t^{1}}^{A} \sum_{j=0}^{n_{s}-1} \\
& \left|\sum_{k=0}^{M_{s}-1} \Phi\left(A, n^{(s+1)}+j M_{s}+k, x^{1}, x^{2}\right)\right| d \mu(x) \\
& :=B^{3} .
\end{aligned}
$$

The method we are going to use in order to discuss $B^{3}$ is the same as we used for the investigation of $B^{1}$. The only difference is that in the situation of $B^{1}$ we used the estimation $\left|D_{\alpha_{2}\left(A, n^{(s+1)}+j M_{s}+k\right)}\left(y^{2}, x^{2}\right)\right| \leq C M_{t^{2}}$ and in the case of $B^{3}$ we use the formula of the Dirichlet kernel $D_{n}$ (1.5) and the estimation $\left|D_{\alpha_{2}\left(A, n^{(s+1)}+j M_{s}+k\right)}\left(y^{2}, x^{2}\right)\right| \leq C M_{A}$. The other steps of this process are the same. That is,

$$
\begin{gathered}
B^{3} \leq C \sum_{t^{1}=0}^{a-1} \sum_{t^{2}=a+c}^{\infty} \int_{J^{2}\left(y^{2}\right)} \sum_{A=a}^{t^{2}-c} \frac{1}{M_{A}} \sum_{s=t^{1}}^{A} M_{t^{1}}^{-\frac{1}{2}} \\
\times\left[\sum_{n_{A}=1}^{m_{A}-1} \sum_{n_{A-1}=0}^{m_{A-1}-1} \cdots \sum_{n_{s+1}=0}^{m_{s+1}-1}\left(M_{t^{1}} M_{A}\right)^{2} M_{s} M_{t^{1}}\right]^{\frac{1}{2}} d \mu\left(x^{2}\right) \\
=C \sum_{t^{1}=0}^{a-1} \sum_{t^{2}=a+c}^{\infty} \sum_{A=a}^{t^{2}-c} \sum_{s=t^{1}}^{A} M_{t^{2}}^{-1} M_{A}^{-1} M_{t^{1}}^{-\frac{1}{2}}\left(M_{A} M_{s}^{-1} M_{t^{1}}^{2} M_{A}^{2} M_{S}\right)^{\frac{1}{2}} \\
\leq C \sum_{t^{1}=0}^{a-1} \sum_{t^{2}=a+c}^{\infty} \sum_{A=a}^{t^{2}-c} \sum_{s=t^{1}}^{A} \frac{\left(M_{A} M_{t^{1}}\right)^{\frac{1}{2}}}{M_{t^{2}}} \\
\leq C \sum_{t^{1}=0}^{a-1} \sum_{t^{2}=a+c}^{\infty} \sum_{A=a}^{t^{2}-c}\left(A-t^{1}+1\right) M_{A}^{\frac{1}{2}} M_{t^{1}}^{\frac{1}{2}} M_{t^{2}}^{-1} \\
\leq C \sum_{t^{1}=0}^{a-1} \sum_{t^{2}=a+c}^{\infty}\left(t^{2}-t^{1}+1\right) M_{t^{1}}^{\frac{1}{2}} M_{t^{2}}^{-\frac{1}{2}} \leq C .
\end{gathered}
$$

This completes the proof. 
In the sequel we step further and with the application of Lemma 3, we prove the main tool with respect to the maximal generalized Marcinkiwicz kernel in order to prove that the maximal operator $t_{*}^{\alpha}:=\sup _{n \in \mathbb{P}}\left|t_{n}^{\alpha} f\right|$ is quasi-local (for the definition of quasi-locality, see e.g. [16, page 262]) and then it is of weak type $\left(L^{1}, L^{1}\right)$.

Lemma 4. Let $u \in G_{m}^{2}, a \in \mathbb{N}, y \in I_{a}\left(u^{1}\right) \times I_{a}\left(u^{2}\right)$. Then we have

$$
\int_{G_{m}^{2}\left(\backslash I_{a}\left(u^{1}\right) \times I_{a}\left(u^{2}\right)\right)} \sup _{n \geq M_{a-c}}\left|M_{n}^{\alpha}(y, x)\right| d \mu(x) \leq C .
$$

Proof. For $t^{1} \leq a-1, t^{2} \geq t^{1}$ and $x \in J_{t^{1}}\left(y^{1}\right) \times J_{t^{2}}\left(y^{2}\right)$ by (1.5) and (2.2) it is clear that

$$
\begin{aligned}
& \left|\Phi\left(A, n^{(s+1)}+j M_{s}+k, y, x\right)\right| \\
& =\left|D_{\alpha_{1}\left(A, n^{(s+1)}+j M_{s}+k\right)}\left(y^{1}, x^{1}\right) D_{\alpha_{2}\left(A, n^{(s+1)}+j M_{s}+k\right)}\left(y^{2}, x^{2}\right)\right| \leq C M_{t^{1}} M_{\min \left(t^{2}, A\right)} .
\end{aligned}
$$

This gives,

$$
\begin{aligned}
& \sum_{t^{1}=0}^{a-1} \sum_{t^{2}=t^{1}}^{\infty} \int_{J_{t^{1}}\left(y^{1}\right) \times J_{t^{2}}\left(y^{2}\right)} \sup _{A \geq a} \sup _{|n|=A} \frac{1}{M_{A}} \sum_{s=0}^{t^{1}} \sum_{j=0}^{n_{s}-1}\left|\sum_{k=0}^{M_{s}-1} \Phi\left(A, n^{(s+1)}+j M_{s}+k, y, x\right)\right| d \mu(x) \\
& \leq C \sum_{t^{1}=0}^{a-1} \sum_{t^{2}=t^{1}}^{\infty} \int_{J_{t^{1}}\left(y^{1}\right) \times J_{t^{2}}\left(y^{2}\right)} \sup _{A \geq a-c} \frac{1}{M_{A}} \sum_{s=0}^{t^{1}} M_{S} M_{t^{1}} M_{\min \left(t^{2}, A\right)} d \mu(x) \\
& \leq C \sum_{t^{1}=0}^{a-1} \sum_{t^{2}=t^{1}}^{a-c} \frac{1}{M_{t^{1}} M_{t^{2}}} \sup _{A \geq a-c} \frac{1}{M_{A}} M_{t^{1}}^{2} M_{t^{2}}+C \sum_{t^{1}=0}^{a-1} \sum_{t^{2}=a-c}^{\infty} \frac{1}{M_{t^{1}} M_{t^{2}}} M_{t^{1}}^{2} \\
& \leq C \sum_{t^{1}=0}^{a-1} \sum_{t^{2}=t^{1}}^{a-c} M_{a}^{-1} M_{t^{1}}+C \sum_{t^{1}=0}^{a-1} \sum_{t^{2}=a-c}^{\infty} M_{t^{1}} M_{t^{2}}^{-1} \leq C .
\end{aligned}
$$

This by equality

$$
M_{n}^{\alpha}(y, x)=\frac{1}{n} \sum_{s=0}^{A} \sum_{j=0}^{n_{s}-1} \sum_{k=0}^{M_{s}-1} \Phi\left(A, n^{(s+1)}+j M_{s}+k, y, x\right)
$$

and by Lemma 3 immediately follows that

$$
\sum_{t^{1}=0}^{a-1} \sum_{t^{2}=t^{1}}^{\infty} \int_{J_{t^{1}}\left(y^{1}\right) \times J_{t^{2}}\left(y^{2}\right)} \sup _{\{n:|n|=A \geq a-c\}}\left|M_{n}^{\alpha}(y, x)\right| d \mu(x) \leq C .
$$

Now, we prove for each $y \in I_{a}\left(u^{1}\right) \times I_{a}\left(u^{2}\right)$ the almost everywhere relation

$$
\begin{aligned}
& G_{m}^{2} \backslash\left(I_{a}\left(u^{1}\right) \times I_{a}\left(u^{2}\right)\right) \\
& \subset\left(\bigcup_{t^{1}=0}^{a-1} \bigcup_{t^{2}=t^{1}}^{\infty} J_{t^{1}}\left(y^{1}\right) \times J_{t^{2}}\left(y^{2}\right)\right) \cup\left(\bigcup_{t^{1}=0}^{a-1} \bigcup_{t^{1}=t^{2}}^{\infty} J_{t^{1}}\left(y^{1}\right) \times J_{t^{2}}\left(y^{2}\right)\right) \\
& =: J^{1}(y) \cup J^{2}(y) .
\end{aligned}
$$


Let $x=\left(x^{1}, x^{2}\right) \in G_{m}^{2} \backslash\left(I_{a}\left(u^{1}\right) \times I_{a}\left(u^{2}\right)\right)$. Then, either $x^{1} \notin I_{a}\left(u^{1}\right)$ or $x^{2} \notin I_{a}\left(u^{2}\right)$ (or both). Say, $x^{1}$ is not element of $I_{a}\left(u^{1}\right)$. Then, $x^{1} \in J_{t^{1}}\left(u^{1}\right)=J_{t^{1}}\left(y^{1}\right)$ for some $t^{1}<a$.

If $x^{2} \in I_{a}\left(u^{2}\right)=I_{a}\left(y^{2}\right)$ and $x^{2} \neq y^{2}$, then $x \in J^{1}(y)$. If $x^{1} \in J_{t^{1}}\left(u^{1}\right)=J_{t^{1}}\left(y^{1}\right)$ and $x^{2}$ is not element of $I_{a}\left(u^{2}\right)=I_{a}\left(y^{2}\right)$, then, $x^{1} \in J_{t^{1}}\left(u^{1}\right)=J_{t^{1}}\left(y^{1}\right)$ and $x^{2} \in J_{t^{2}}\left(u^{2}\right)=J_{t^{2}}\left(y^{2}\right)$ for some $t^{1}, t^{2}<a$.

For $t^{2} \geq t^{1}$ we have $x \in J^{1}(y)$ and for $t^{1} \geq t^{2}$ we have $x \in J^{2}(y)$. This procedure can be done if $x^{1}, x^{2}$ different from $y^{1}$ and $y^{2}$ respectively. The set of the points $x=\left(x^{1}, x^{2}\right)$, where either $x^{1}=y^{1}$ or $x^{2}=y^{2}$ is a zero measure set, so this can be supposed and the a.e. relation $G_{m}^{2} \backslash\left(I_{a}\left(u^{1}\right) \times I_{a}\left(u^{2}\right)\right) \subset J^{1}(y) \cup J^{2}(y)$ is proved for each $y \in I_{a}\left(u^{1}\right) \times I_{a}\left(u^{2}\right)$. Therefore, the proof of Lemma 4 is complete.

Corollary 1. Let $y \in G_{m}, n \in \mathbb{P}$. Then, $\left\|M_{n}^{\alpha}(y, \cdot)\right\|_{1} \leq C$.

Proof. By Lemma 4, we have $\int_{G_{m}^{2} \backslash\left(I_{|n|}\left(y^{1}\right) \times I_{|n|}\left(y^{2}\right)\right)}\left|M_{n}^{\alpha}(y, x)\right| d \mu(x) \leq C$. Besides, by the conditions (2.1) and (2.2)

$$
\left|M_{n}^{\alpha}(y, x)\right| \leq \frac{1}{n} \sum_{k=0}^{n-1} D_{\alpha_{1}(|n|, k)}\left(y^{1}, x^{1}\right) D_{\alpha_{2}(|n|, k)}\left(y^{2}, x^{2}\right) \leq C \frac{1}{n} \sum_{k=0}^{n-1} M_{|n|} M_{|n|} \leq C M_{|n|}^{2} .
$$

Consequently,

$$
\int_{I_{|n|}\left(y^{1}\right) \times I_{|n|}\left(y^{2}\right)}\left|M_{n}^{\alpha}(y, x)\right| d \mu(x) \leq C .
$$

Hence, $\left\|M_{n}^{\alpha}(y, \cdot)\right\| \leq C$.

Now, let's check the quasi-locality of the maximal operator $t_{*}^{\alpha}$ is quasi-local (for the definition of the quasi-locality, see e.g. [16, page 262]).

Lemma 5. Let $f \in L^{1}\left(G_{m}^{2}\right)$ such that $\operatorname{supp} f \subset I_{a}\left(u^{1}\right) \times I_{a}\left(u^{2}\right), \int f d \mu(x)=0$ for some $u \in G_{m}^{2}$ and $a \in \mathbb{N}$. Then, $\int_{G_{m}^{2} \backslash I_{a}\left(u^{1}\right) \times\left(I_{a}\left(u^{2}\right)\right)} t_{*}^{\alpha} f(x) d \mu(x) \leq C\|f\|_{1}$.

Proof. If $|n| \leq a-c$ for some fixed constant $c>0$ depending only on $\alpha_{1}$ and $\alpha_{2}$, then we have by (4) that $\alpha_{1}(|n|, k), \alpha_{2}(|n|, k)<M_{a}$ for every $k<n$. Consequently, the kernel $M_{n}^{\alpha}(y, x)$, which is a linear combination of the product of Vilenkin-like functions $\chi_{k}$ with $k<M_{a}$, which is $\mathcal{A}_{a}$ measurable. This implies that

$$
t_{n}^{\alpha} f(y)=\int_{I_{a}^{2}(u)} f(x) M_{n}^{\alpha}(x, y) d \mu(x)=M_{n}^{\alpha}(y) \int_{I_{a}\left(u^{1}\right) \times I_{a}\left(u^{2}\right)} f(x) d \mu=0 .
$$


That is, $|n| \geq a-c$ can be supposed. By the theorem of Fubini, by Lemma 4 and by the fact that for kernel $\left|M_{n}^{\alpha}(y, x)\right|=\left|M_{n}^{\alpha}(x, y)\right|$ we get,

$$
\begin{aligned}
& \int_{G_{m}^{2} \backslash I_{a}^{2}(u)} t_{*}^{\alpha} f \\
& =\int_{G_{m}^{2} \backslash I_{a}^{2}(u)} \sup _{|n| \geq a-c}\left|t_{n}^{\alpha} f\right| d \mu(x) \\
& =\int_{G_{m}^{2} \backslash I_{a}^{2}(u)} \sup _{|n| \geq a-c}\left|\int_{I_{a}^{2}(u)} f(x) M_{n}^{\alpha}(y, x) d \mu(x)\right| d \mu(y) \\
& \leq \int_{I_{a}^{2}(u)}|f(x)| \int_{G_{m}^{2} \backslash I_{a}^{2}(u)} \sup _{|n| \geq a-c}\left|M_{n}^{\alpha}(y, x)\right| d \mu(y)\left|d \mu(x) \leq C \int_{I_{a}^{2}(u)}\right| f(x)|d \mu(x)=C| \mid f \|_{1} .
\end{aligned}
$$

This completes the proof of Lemma 5.

Theorem 1. The operator $t_{*}^{\alpha}$ is of weak type $\left(L^{1}, L^{1}\right)$ and it is also of type $\left(L^{p}, L^{p}\right)$ for all $1<p \leq \infty$.

Proof. Now, we know that the operator $t_{*}^{\alpha}$ is of type $\left(L^{\infty}, L^{\infty}\right)$ which is given by Corollary 1 and it is quasi-local by Lemma 5 . Consequently, to prove that operator $t_{*}^{\alpha}$ is of weak type $\left(L^{1}, L^{1}\right)$ is nothing else but to follow the standard argument (see e.g. [16]). Finally, the interpolation lemma of the Marcinkiwicz gives that it is also of type $\left(L^{p}, L^{p}\right)$ for all $1<p \leq \infty$. Consequently, by the above written by standard argument, we have the following theorem.

Theorem 2. Let $\alpha$ satisfy conditions (2.1) and (2.2). Then, we have $t_{n}^{\alpha} \rightarrow f$ for each $f \in L^{1}\left(G_{m}^{2}\right)$ a.e. with respect to every bounded Vilenkin-like system.

Proof. The proof of Theorem 2 is just a standard consequence of the fact that the maximal operator $t_{*}^{\alpha}$ is of weak type $\left(L^{1}, L^{1}\right)$, the fact that it holds for each twodimensional Vilenkin-like polynomial (linear combinations of $\chi_{k}\left(x^{1}\right) \chi_{n}\left(x^{2}\right)$ ) and the fact that the set of two-dimensional Vilenkin-like polynomials is dense in $L^{1}\left(G_{m}^{2}\right)$. This density property comes from the behavior of the one dimensional kernel function $D_{M_{n}}$. That is, it is either $M_{n}$ or zero.

Finally, we give an application of Theorem 2. Before this a corollary is given:

Corollary 2. Let $\left(a_{n}\right)$ be a lacunary sequence of positive reals, i.e. $a_{n+1} \geq a_{n} q$ for some $q>1(n \in \mathbb{N})$ and $\alpha$ satisfy conditions (3) and $\alpha_{j}(n, k) \leq C a_{n}\left(k<a_{n}, j=1,2\right)$ (modified version of condition (2.2). Then for every integrable function $f \in L^{1}\left(G_{m}^{2}\right)$ we have

$$
\frac{1}{a_{n}} \sum_{k=0}^{a_{n}-1} S_{\alpha_{1}(n, k), \alpha_{2}(n, k)} f(x) \rightarrow f(x)
$$

for a.e. $x \in G_{m}^{2}$. 
Proof. The proof of this corollary runs as follows. Let $b_{n}$ be defined as $M_{b_{n}-1} \leq$ $a_{n}<M_{b_{n}}$ (that is, $\left.b_{n}=\left|a_{n}\right|+1\right)$ and

$$
\tilde{\alpha}_{j}\left(b_{n}, k\right)=\left\{\begin{array}{ll}
\alpha(n, k), & \text { for } 0 \leq k<a_{n}, \\
k, & \text { if } a_{n} \leq k<M_{b_{n}}
\end{array} \quad(j=1,2) .\right.
$$

Then, $\tilde{\alpha}$ satisfies conditions (2.1) (trivially) and (2.2) since for $k<a_{n}, \tilde{\alpha}_{j}\left(b_{n}, k\right)=$ $\alpha_{j}(n, k) \leq C a_{n} \leq C M_{b_{n}}$. By Theorem 2 it follows that for the maximal operator $t_{*}^{\tilde{\alpha}} f:=\sup \left|t_{n}^{\tilde{\alpha}} f\right|$ we have $\mu\left\{t_{*}^{\tilde{\alpha}} f \geq \lambda\right\} \leq C\|f\|_{1} / \lambda$ for all $f \in L^{1}\left(G_{m}^{2}\right)$ and $\lambda>0$. Since

$$
\frac{1}{a_{n}} \sum_{k=0}^{a_{n}-1} S_{\alpha_{1}(n, k), \alpha_{2}(n, k)} f=\frac{M_{b_{n}}}{a_{n}} \frac{1}{M_{b_{n}}} \sum_{k=0}^{M_{b_{n}}-1} S_{\tilde{\alpha}_{1}\left(b_{n}, k\right), \tilde{\alpha}_{2}\left(b_{n}, k\right)} f-\frac{M_{b_{n}}}{a_{n}} \frac{1}{M_{b_{n}}} \sum_{k=a_{n}}^{M_{b_{n}}-1} S_{k, k} f
$$

and consequently, $\left|t_{a_{n}}^{\alpha} f\right| \leq C\left|t_{M_{b_{n}}}^{\tilde{\alpha}} f\right|+C\left|t_{M_{b_{n}}}^{\text {id }} f\right|+C\left|t_{a_{n}}^{\text {id }} f\right|$, then (id denotes the "identical function", i.e. $\operatorname{id}(n, k)=(k, k)) t_{*}^{\alpha} f \leq C t_{*}^{\tilde{\alpha}} f+C t_{*} f$. The ordinary maximal Marcinkiewicz operator is of weak type $\left(L^{1}, L^{1}\right)$ (see e.g. [6]) and this by standard argument [16] completes the proof of this corollary.

In the sequel we give an application of the Corollary above. The triangular partial sums of the 2-dimensional Fourier series and the triangular Dirichlet kernels (with respect to the Vilenkin-like system $\chi$ ) are defined as

$$
S_{k}^{\triangle} f\left(x^{1}, x^{2}\right):=\sum_{i=0}^{k-1} \sum_{j=0}^{k-i-1} \hat{f}(i, j) \chi_{i}\left(x^{1}\right) \chi_{j}\left(x^{2}\right), \quad D_{k}^{\triangle}\left(x^{1}, x^{2}\right):=\sum_{i=0}^{k-1} \sum_{j=0}^{k-i-1} \chi_{i}\left(x^{1}\right) \chi_{j}\left(x^{2}\right) .
$$

The Fejér means of the triangular partial sums of the two-dimensional integrable function $f$ (see e.g. [11]) are

$$
\sigma_{n}^{\triangle} f:=\frac{1}{n} \sum_{k=0}^{n-1} S_{k}^{\triangle} f
$$

For the trigonometric system Herriot proved [12] the a.e. (and norm) convergence $\sigma_{n}^{\triangle} f \rightarrow f\left(f \in L^{1}\right)$. His method can not be adopted for the Vilenkin system, since for the time being there is no kernel formula available for these systems. The first result in this a.e. convergence issue of triangular means is due to Goginava and Weisz [11]. They proved for the Walsh-Paley system and each integrable function the a.e. convergence relation $\sigma_{2^{n}}^{\triangle} f \rightarrow f$. This result for the whole sequence of the triangular mean operators in the Walsh case is given by the first author [9]. In the Vilenkin situation there is nothing proved yet. By the corollary above, (Corollary 2) we prove for bounded Vilenkin-like systems:

Theorem 3. For every lacunary sequence $\left(a_{n}\right)$ (that is, $a_{n+1} \geq q a_{n}, q>1$ ) we have the a.e. convergence $\sigma_{a_{n}}^{\triangle} f \rightarrow f$ for each $f \in L^{1}\left(G_{m}^{2}\right)$. 
To demonstrate the proof of this, see some calculations below [9] between the triangle and the one dimensional Dirichlet kernels.

$$
\begin{aligned}
& K_{n}^{\triangle}\left(y^{1}, y^{2}, x^{1}, x^{2}\right)=\frac{1}{n} \sum_{k=0}^{n-1} D_{k}^{\triangle}\left(y^{1}, y^{2}, x^{1}, x^{2}\right)=\frac{1}{n} \sum_{k=1}^{n-1} \sum_{i=0}^{k-1} \sum_{j=0}^{k-i-1} \chi_{i}\left(y^{1}, x^{1}\right) \chi_{j}\left(y^{2}, x^{2}\right) \\
& =\frac{1}{n} \sum_{k=1}^{n-1} \sum_{i=0}^{k-1} \chi_{i}\left(y^{1}, x^{1}\right) D_{k-i}\left(y^{2}, x^{2}\right)=\frac{1}{n} \sum_{k=1}^{n-1} \sum_{i=1}^{k} \chi_{k-i}\left(y^{1}, x^{1}\right) D_{i}\left(y^{2}, x^{2}\right) \\
& =\frac{1}{n} \sum_{i=1}^{n-1} \sum_{k=i}^{n-1} \chi_{k-i}\left(y^{1}, x^{1}\right) D_{i}\left(y^{2}, x^{2}\right)=\frac{1}{n} \sum_{i=1}^{n-1} D_{n-i}\left(y^{1}, x^{1}\right) D_{i}\left(y^{2}, x^{2}\right) \\
& =\frac{1}{n} \sum_{i=1}^{n-1} D_{i}\left(y^{1}, x^{1}\right) D_{n-i}\left(y^{2}, x^{2}\right)
\end{aligned}
$$

which is a generalized Marcienkiwicz kernel discussed in this paper (see Corollary 8:

$\left.\alpha_{1}(|n|, k)=k, \alpha_{2}(|n|, k)=a_{n}-k\right)$.

Acknowledgement. The authors are indebted to the anonymous referee for his/her valuable help.

\section{REFERENCES}

[1] G. Agaev, N. Vilenkin, G. Dzhafarli, and A. Rubinstein, Multiplicative Systems of Functions and Harmonic Analysis on 0-dimensional Groups. Baku: ELM, 1981.

[2] I. Blahota and G. Gát, "Almost everywhere convergence of Marcinkiewicz means of Fourier series on the group of 2-adic integers," Studia Mathematica, vol. 191, no. 3, pp. 215-222, 2009, doi: 10.4064/sm191-3-3.

[3] M. Dyachenko, " $(C, \alpha)$-summability of multiple trigonometric Fourier series," Soobshch. Akad. Nauk Gruzin. SSR, vol. 131, no. 2, pp. 261-263, 1988.

[4] G. Gát, "On almost even arithmetical functions via orthonormal systems on Vilenkin groups," Acta Arith., vol. 60, no. 2, pp. 105-123, 1991, doi: 10.4064/aa-60-2-105-123. [Online]. Available: https://doi.org/10.4064/aa-60-2-105-123

[5] G. Gát, "Orthonormal systems on Vilenkin groups," Acta Math. Hungar., vol. 58, no. 1-2, pp. 193 198, 1991, doi: 10.1007/BF01903559. [Online]. Available: https://doi.org/10.1007/BF01903559

[6] G. Gát, "Convergence of Marcinkiewicz means of integrable functions with respect to twodimensional Vilenkin systems," Georgian Math. J., vol. 11, no. 3, pp. 467-478, 2004.

[7] G. Gát, "On almost everywhere convergence and divergence of Marcinkiewicz-like means of integrable functions with respect to the two-dimensional Walsh system," J. Approx. Theory, vol. 164, no. 1, pp. 145-161, 2012, doi: 10.1016/j.jat.2011.09.010. [Online]. Available: https://doi.org/10.1016/j.jat.2011.09.010

[8] G. Gát, "Marcinkiewicz-like means of two dimensional Vilenkin-Fourier series," Publ. Math. Debrecen, vol. 89, no. 3, pp. 331-346, 2016, doi: 10.5486/PMD.2016.7580. [Online]. Available: https://doi.org/10.5486/PMD.2016.7580

[9] G. Gát, "Almost everywhere convergence of Fejér means of two-dimensional triangular Walsh-Fourier series," J. Fourier Anal. Appl., vol. 24, no. 5, pp. 1249-1275, 2018, doi: 10.1007/s00041-017-9566-2. [Online]. Available: https://doi.org/10.1007/s00041-017-9566-2 
[10] U. Goginava, "Almost everywhere summability of multiple Walsh-Fourier series," J. Math. Anal. Appl., vol. 287, no. 1, pp. 90-100, 2003, doi: 10.1016/S0022-247X(03)00503-1. [Online]. Available: https://doi.org/10.1016/S0022-247X(03)00503-1

[11] U. Goginava and F. Weisz, "Maximal operator of the Fejér means of triangular partial sums of two-dimensional Walsh-Fourier series," Georgian Math. J., vol. 19, no. 1, pp. 101-115, 2012, doi: 10.1515/gmj-2012-0004. [Online]. Available: https://doi.org/10.1515/gmj-2012-0004

[12] J. Herriot, "Nörlund summability of multiple Fourier series," Duke Math. J., vol. 11, pp. 735-754, 1944. [Online]. Available: http://projecteuclid.org/euclid.dmj/1077472809

[13] E. Hewitt and K. Ross, Abstract harmonic analysis. Vol. I, 2nd ed., ser. Grundlehren der Mathematischen Wissenschaften [Fundamental Principles of Mathematical Sciences]. Springer-Verlag, Berlin-New York, 1979, vol. 115, structure of topological groups, integration theory, group representations.

[14] J. Marcinkiewicz, "Quelques théorèmes sur les séries orthogonales," Ann. Soc. Polon. Math., vol. 16, pp. 85-96, 1937.

[15] J.-L. Mauclaire, Intégration et théorie des nombres, ser. Travaux en Cours. [Works in Progress]. Hermann, Paris, 1986, with a preface by S. Iyanaga.

[16] F. Schipp, W. R. Wade, and P. Simon, Walsh series. Adam Hilger, Ltd., Bristol, 1990, an introduction to dyadic harmonic analysis, With the collaboration of J. Pál.

[17] F. Weisz, "Convergence of double Walsh-Fourier series and Hardy spaces," Approx. Theory Appl. (N.S.), vol. 17, no. 2, pp. 32-44, 2001, doi: 10.1023/A:1015553812707. [Online]. Available: https://doi.org/10.1023/A:1015553812707

[18] L. Zhizhiasvili, "Generalization of a theorem of Marcinkiewicz," Akad. Nauk USSR Ser Mat., vol. 32, pp. 1112-1122, 1968.

Authors' addresses

\section{György Gát}

G. Gát, Institute of Mathematics, University of Debrecen, H-4002 Debrecen, Pf. 400, Hungary

E-mail address: gat.gyorgyescience. unideb.hu

\section{Anteneh Tilahun}

Anteneh Tilahun, Institute of Mathematics, University of Debrecen, Hungary

E-mail address: antenehescience. unideb.hu 